\title{
Ion Channel Focusing in FEL Wigglers
}

\author{
John Vetrovec \\ Rockwell International, Rocketdyne Division \\ 6633 Canoga Ave. \\ Canoga Park, Calif. 91304, (818) 700-4875
}

\section{Abstract}

Accumulation of ions inside FEL wigglers can significantly alter electron beam transport and lead to a reduction of laser gain. This is of particular importance to devices driven by RF accelerators with continuous operation and with long wigglers. We show that while the mechanism for ion channel formation is the same as in electron beam storage rings, the problem appears less severe because the electron beam makes only a single pass through the systcm. In addition, the electric field of the beam together with the magnetic ficld of the wiggler cause an $E x B$ drift which can provide effective mechanism for ion removal.

\section{INTRODUCTION}

The Ground Based Free Electron Laser Technology Integration Experiment (GBFEL-TIE) planned for White Sands, New Mexico, has a continuously operating RF accelerator. Electron beam transport in this system is subject to ion trapping often found in clectron storage rings. The probIem appears to be most severe in the wiggler where spatial constraints impede vacuum pumping and reduce effectiveness of ion clearing electrodes. In addition, ions have been suspected to accumulate inside wigglers by magnetic mirror effect. Even a small amount of ions trapped inside storage ring beams is highly undesirable because they cause a reduction of beam lifetime and brightness. This problem is unique to cyclic devices where effects of repeated interactions between the beam and trapped ions are cumulative. Ion population in storage rings is controlled by operating the beamlinc at a very high vacuum, typically in the range of $10^{-10} \mathrm{Torr}$, and by ion extraction electrodes.

Typical FEL has a single pass electron beam system and, therefore, is not susceptible to cumulative effects. However, for practical reasons the GBFEL-TIE beamline is designed to operate at a higher pressure (about $10^{-7}-10^{-8}$ Torr) resulting in higher ionization rates. In addition, the wiggler, which is $15 \mathrm{~m}$ long, has a small beam aperture with poor side access for vacuum pumping. That leads not only to additional increase in pressure, but also makes ion extraction electrodes less effective. In absence of efficient ion loss mechanism, a dense ion channel may form inside the wiggler, potentially causing focusing mismatch and emittance growth. As a result, the FEL gain could be compromised. This work investigates criteria for beam neutralization, ion motion in the combined wiggler field and electrostatic potential well, and the impact of beam neutralization on transport as applicd to the $15 \mathrm{~m}$ GBFEL-TIE wiggler. Our analysis indicates that the magnetic mirror trapping may not be very strong, and that the ions are subject to ExB drift which may provide an effective ion removal mechanism. This result is also supported by computer models.

\section{ION CHANNEL FORMATION}

An clectron beam propagating through the vacuum tube collides with and ionizes molecules of the residual gas. Electrons born in the ionization process are ejected while the ions are trapped by the elcctric field of the beam. In the absence of an efficient loss mechanism the ion plasma density in the channel can exceed that of the background gas. An electron beam of average current $I_{\text {avg }}$ propagating through background gas of density $n_{o}$ produces ions at a rate

$$
\frac{d N_{i}}{d t}=\frac{I_{\text {avg }}}{e} \sigma_{01} n_{o}
$$

where $e$ is the elementary charge and $\sigma_{01}$ is the ionization cross section. At the $100 \mathrm{MeV}$ electron beam energy the cross-section does not vary significantly for the gases of interest [1] and the value of $1.5 \times 10^{-18} \mathrm{~cm}^{-2}$ has been used in all calculations. With the background gas at $10^{-8}$ Torr pressure and $0.23 \mathrm{~A}$ average current the ion production rate in the GBFEL-TIE wiggler can be as high as $7.6 \times 10^{9}$ ions $\mathrm{cm}^{-1}$ $\sec ^{-1}$.

In the absence of a magnetic field the transverse motion of an ion of charge ze and mass $M$ immersed in the beam is described by

$$
\ddot{r}-\frac{z e}{M}\left[E_{i}+E_{b} P(t)\right]=0
$$

where $r$ is the radial coordinate, $t$ is time, $E_{i}$ and $E_{b}$ are respectively the instantaneous electric fields produced by the ion and electron space charges, and $P(t)$ is the micropulse format function. To simplify this analysis, we assume that both the electron beam and the trapped ions have a uniform transverse density distribution. Let $b$ be the beam radius, $T_{I}$ the beam bunch length in scconds, $F_{I}$ the bunch frequency, $<z>$ the average degree of ionization of the ion plasma, and $N_{i}$ the ion line density. Then, $E_{i}(r)=\left\langle z>e N_{i} r /\left(2 \pi \epsilon_{i} b^{2}\right)\right.$ and $E_{b}(r)=-I_{a r g} r /\left(2 \pi \epsilon_{\alpha} c \beta b^{2} F_{1} T_{1}\right)$ where $\epsilon_{o}$ is the permittivity of free space, $c$ the speed of light, and $\beta$ the relativistic factor.

To find the limiting ion line density we note that if the beam is completely neutralized, ion motion is barely stable. From eq. (2) it is seen that this happens when $<z>e N_{i}=I_{\text {avg }} /(c \beta)$, i.e., when the ion line charge is same as the time averaged line charge of the electron beam. We define the average fractional neutralization $f_{\text {avg }}$ as the ratio of the ion line density and the average electron line density, i.e., $f_{a r g}=\langle z\rangle e N_{i} c \beta / I_{\text {arg }}$. When the beam is completely neutralized, $f_{\text {avg }}=1$. This translates to a line 
density $N_{i}=4.8 \times 10^{7} \mathrm{~cm}^{-1}$, which is about an order of magnitude higher than the density of the background neutral gas at $10^{-8}$ 'Torr. It is also useful to define the instantaneous neutralization $f_{1}$ as the ratio of the ion line density and the instantancous electron line charge, i.e., $f_{1}=\langle z\rangle e N_{i} c \beta$ $T_{1} F_{1} / l_{\text {avg }}$. Using the GBFEL-TIE operating parameters, $F_{1}$ $=54 \mathrm{MHz}$ and $T_{1}=18 \mathrm{ps}$, we get $f_{1}=0.00097 f_{\text {avg }}$.

Except for well neutralized beams, the kinetic energy of a newly born ion is not sufficient to climb out of the potential well presented by the beam space charge and the ion is trapped. However, as the ions accumulate, the potential well becomes more shallow and some of the previously trapped ions escape. Again, assuming a uniform beam density, the time dependent potential inside the beam can be expressed as

$$
U(r, t)=\frac{1}{4 \pi \epsilon_{o}}\left[<z>e N_{i}(t)-\frac{I_{\text {arg }}}{c \beta}\right]\left(1-\frac{r^{2}}{b^{2}}\right)
$$

Combining eq. (1) and (3) it can be shown that the time dependent average neutralization fraction is then

$$
f_{a r g}(t)=\left[1+\left(c \beta \sigma_{01} n_{d} t\right)^{-1}\right]^{-1}
$$

This means that at $10^{-8}$ Torr (and in absence of other ion loss mechanism) the electron beam would be $50 \%$ neutralized in about $60 \mathrm{~ms}$ and $90 \%$ neutralized in about $650 \mathrm{~ms}$. Evidently, unless ions are efficiently removed, continuous operation of the accelerator leads to a fully neutralized beam.

\section{EFFECTS ON ELECTRON BEAM TRANSPORT}

Dense ion channel present in the wiggler partially neutralizes the electron beam which upsets the Lorentzian cancellation of beam self fields and the beam tends to focus. This changes the acceptance of the wiggler and produces a mismatch to the injection optics. In particular, the beam envelope inside the wiggler satisfies the equation [2]

$$
\frac{d^{2} b}{d s^{2}}+k_{\beta}^{2} b-\frac{\epsilon_{m i s}^{2}}{b^{3}}-\frac{K_{f}}{b}=0
$$

where $s$ is the beam propagation path, $k_{\beta}$ is the wiggler betatron wave number, $\epsilon_{m s}$ is the rms emittance, and $K_{f}$ is the generalized perveance

$$
K_{f} \equiv \frac{2 I_{\text {avg }} r_{c}\left(1-\beta^{2}-f_{1}\right)}{e c \beta^{3} \gamma}
$$

with $r_{c}$ being the classical particle radius, and $\gamma$ the relativistic factor. Equation (5) has been numerically solved for various levels of neutralization and the results are plotted in Figure 1. As the neutralization sets in, the envelope of the beam starts to oscillate. Changing the cross section of the electron beam reduces the overlap between the electron and the FEL laser beam, which results in a loss of FEL gain. At $100 \%$ neutralization, the rms beam radius drops to about $65 \%$ of its nominal value which translates to well over $50 \%$ loss of FEL power. This problem is potentially correctable by changing the setting on wiggler injection optics.

In the simple analysis above it is assumed that both the beam and ion channel densities are uniform. Such simplifica-

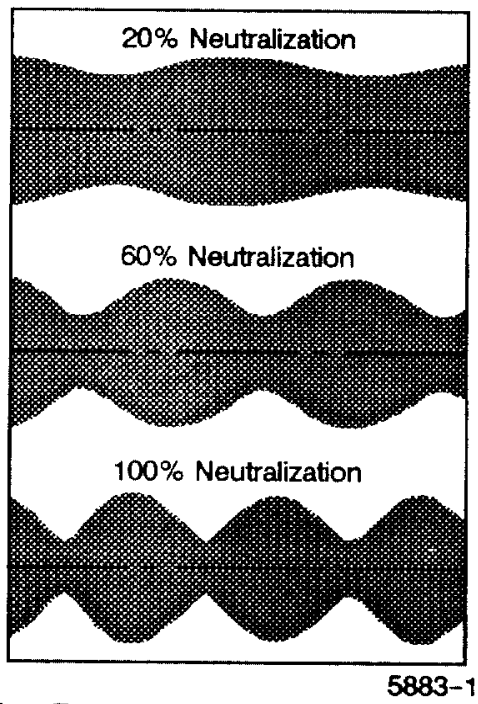

Figure 1. Beam envelope oscillations caused by neutralization driven mismatch into wiggler focusing channel

tion is highly optimistic for realistic electron beams. Moreover, there is little guarantee that the spatial distributions of the beam and the ions are even similar. As a result, the trapped ion plasma could present the electron beam with a highly nonlinear focusing channel. Such conditions are known to produce emittance growth [3]. While the analysis of this phenomenon is beyond the scope of this article, it is recognized that such effects could be of overriding importance [4].

\section{ION LOSS BY EXB DRIFI}

The magnetic field of the wiggler has a profound effect on the motion of trapped ions. First, we note from eq. (2) that the ions execute transverse oscillations at a frequency roughly an order of magnitude lower than the beam bunch frequency. So a test ion reacts only to the time averaged electric field of the combined electron and ion plasma space charge. At the edge of the beam this field has a maximum value $E_{\text {avg }}$ of about $28 \mathrm{kV} / \mathrm{m}$. In the wiggler plane an ion born at a location $x_{o}$ is subjected to an $E x B$ drift

$$
v_{E}=\frac{E_{\text {avg }}}{B_{y}} \frac{x_{o}}{b}
$$

where $B_{y}$ is the local magnetic field which is assumed to be roughly constant over one cyclotron gyration, Figure 2.

This simple model is verified by numerical simulations using a fully 3-dimensional model of magnetic field and particle transport, with a temporal resolution sufficient to track individual $18 \mathrm{ps}$ micropulses of the beam. A sample plot of computed ion trajectory presented in Figure 3 exhibits the characteristic cycloid shape of a particle subjected to ExB drift. The orientation of the cycloid flips as the magnetic field switches polarity. It is important to note that in a single gyration the ion generally remains in a constant magnetic field which validates our simple analysis.

In a wiggler field of $0.5 \mathrm{~T}$ an ion born at the edge of the beam drifts at an average velocity of $5.6 \times 10^{4} \mathrm{~m} / \mathrm{s}$. So the av- 


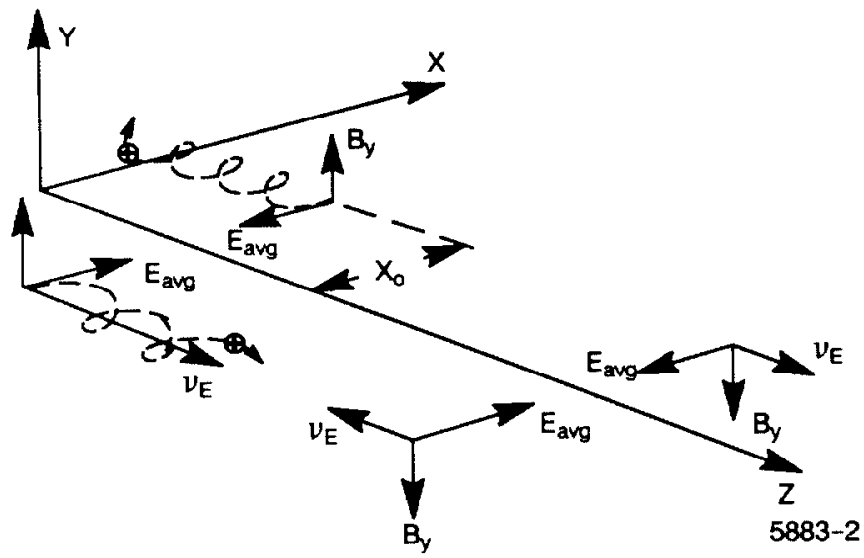

Figure 2. $E X B$ drifts in wiggler field

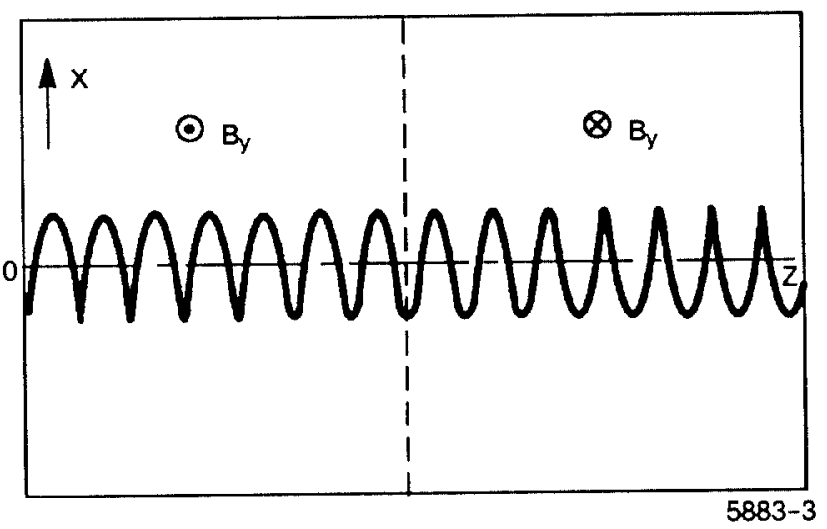

Figure 3. Computer simulation of ion drift

erage ion lifetime $\tau_{i}$ in a wiggler $15 \mathrm{~m}$ long is about $130 \mu \mathrm{s}$. At $10^{-8}$ Torr background pressure this suggests an ion line density $N_{i}=\left(d N_{i} / d t\right) \tau_{i}$ of about $1 \times 10^{6}$ ions $/ \mathrm{cm}$ which translates to average neutralization $f_{\text {avg }}$ of $0.2 \%$. Admittedly, this kind of analysis is exceedingly optimistic. Ions born closer to the beam center see a lower electric field and those born between wiggler poles see a reduced magnetic field.
Also, as the ions start to accumulate, the electric field of the beam is partially canceled. In either case, the drift velocity is reduced. To account for such nonoptimistic situations we estimated the average drift velocity to be a factor of 10 lower then above. Happily, this still translates to a relatively low neutralization of $2 \%$.

Despite the fact that electric and the magnetic field have significant spatial variations, our computer simulations failed to detect ion trapping by magnetic mirror effects as seen by our colleagues [5]. We intend to perform a more thorough investigation of this phenomenon in the future.

\section{CONCLUSION}

We presented our initial analysis of the trapped ion problem expected in the GBFEL-TIE wiggler. In the absence of an effective ion removal mechanism, the electron beam is fully neutralized. While catastrophic beam pinching does not take place, the focusing mismatch could significantly reduce FEL power. In addition, emittance growth is strongly suspected and warrants further study. However, the $E x B$ drift promises to be very effective for removing of trapped ions and reducing the neutralization to a tolerable level. Additional work is needed to to analyze this phenomenon under all operational conditions.

\section{REFERENCES}

1. F. F. Rieke and W. Prepejchal, "Ionization Cross-Sections of Gaseous Atoms and Molecules for High Energy Electrons and Positrons," Phys. Rev. A, Vol. 6, No.4, 1972.

2. J. D. Lawson, "Space Charge Optics," in the "Applied Charged Particle Optics," part C, ed. by Septier, Academic Press, New York, 1983.

3. J. D. Lawson, "The Physics of Charge Particle Beams," Clarendon Press, Oxford, 1988, Sect. 2.2 and 4.4.

4. L. Thode, LANL, personal communications, September 1989.

5. Y. H. Chin, LBL, personal communications, March 1990. 